\title{
Touch-cure polymerization at the composite cement-dentin
}

\section{interface}

Kumiko YOSHIHARA ${ }^{1,2}$, Noriyuki NAGAOKA 3 , Yasuhiko BENINO ${ }^{4}$, Akiko NAKAMURA 5 , Toru $H_{A R A^{5}}$, Yukinori MARUO ${ }^{6}$, Yasuhiro YOSHIDA ${ }^{7}$, and Bart VAN MEERBEEK ${ }^{8}$

${ }^{1}$ National Institute of Advanced Industrial Science and Technology (AIST), Health and Medical Research Institute, 2217-14 Hayashi-cho, Takamatsu, Kagawa 761-0395, Japan

${ }^{2}$ Okayama University, Graduate School of Medicine, Dentistry and Pharmaceutical Sciences, Department of Pathology \& Experimental Medicine, 2-5-1 Shikata-cho, Kita-ku, Okayama 700-8558, Japan

${ }^{3}$ Okayama University Dental School, Advanced Research of Center for Oral and Craniofacial Science, 25-1 Shikata-cho, Kita-ku, Okayama 700-8558, Japan

${ }^{4}$ Okayama university, Graduate School of Environmental and life Science, 1-1-1 Tsushima-naka, Kita-ku, Okayama 700-8530, Japan

${ }^{5}$ National Institute for Materials Science (NIMS), 1-2-1 Sengen, Tsukuba, Ibaraki 305-0047, Japan

${ }^{6}$ Department of Occlusion and Removable Prosthodontics, Okayama University Hospital, 2-5-1 Shikatacho, Kita-ku, Okayama 700-8558, Japan

${ }^{7}$ Hokkaido University, Faculty of Dental Medicine, Department of Biomaterials and Bioengineering, Kita 13, Nishi 7, Kita-ku, Sapporo, Hokkaido 060-8586, Japan

${ }^{8} \mathrm{KU}$ Leuven (University of Leuven), Department of Oral Health Research, BIOMAT \& UZ Leuven (University Hospitals Leuven), Dentistry, Kapucijnenvoer 7, 3000 Leuven, Belgium

Keywords: Cement; Dentin; Electron microscopy; Infrared spectroscopy; Adhesives Words Number: 3194 


\section{ABSTRACT}

Ceramic restorations are often adhesively luted onto the tooth prep. The so-called 'touch-cure' concept was developed to yield optimum polymerization of composite cement at the restoration-cement-tooth interface for immediate bond stabilization. Though this touch cure is theorized to initiate polymerization at the interface when the accelerator in the primer makes contact with the cement, this process has not yet been proven. This study aimed to elucidate the mechanism of touch cure by measuring the degree of conversion (DC) of composite cement applied with/without an accelerator-containing tooth primer (TP) versus an accelerator-free primer using real-time Fourier-transform infrared spectroscopy (RT-FTIR) and attenuated total reflection (ATR)-FTIR. Interfacial bond strength was measured in shear mode, the accelerator composition confirmed by X-ray fluorescence analysis (XRF), and the interfacial interaction of TP and composite cement with dentin investigated by X-ray diffraction (XRD), focused-ion-beam scanning electron microscopy (FIB-SEM) with 3D interface reconstruction, and transmission electron microscopy (TEM). RT/ATR-FTIR revealed the significantly highest DC when the composite cement was applied with the accelerator-containing primer. XRF disclosed a vanadium compound as novel chemical accelerator within TP, instead of a classic chemical curing initiator system, to set off touch cure as soon the cement contacts the beforehand applied primer. Despite TP contains the acidic functional monomer 10-MDP for adhesion to tooth tissue, touch cure using the accelerator-containing TP combined the fastest/highest DC with the highest bond strength. FIB-SEM and TEM confirmed tight interfacial interaction at dentin with submicron hybridization along with stable 10-MDP_Casalt nano-layering. 


\section{Introduction}

Ceramic restorations are often used in cosmetic dentistry in line with the high demand for aesthetic restorations. Advantageously, they can be manufactured efficiently using advanced CAD-CAM techniques. Enabling tooth tissue-preserving restorations, ceramic restorations can adhesively be luted using primer/adhesive-assisted or self-adhesive composite cements. Composite cements most effectively polymerize upon light-curing. However, light irradiation is attenuated in case of thick restorations fabricated of dense ceramics, by which dual-cure composite cements are most often used. Besides bonding effectiveness, polymerization efficiency and related mechanical properties co-determine luting efficiency (De Souza et al., 2015).

Several initiators have been developed to cure resin-based dental materials. Dibenzoyl peroxide (BPO) combined with a tertiary amine is the most commonly used polymerization initiator of dental methyl methacrylates (MMAs) (Achilias et al., 2004; Kwon et al., 2012). Although this BPO/tertiary amine system works well, it is less effective when self-etch primerassisted and self-adhesive composite cements are employed; they contain acidic functional monomers that react with tertiary amine, forming salts instead of BPO-amine free radicals (Kwon et al., 2012). To overcome this drawback, BPO/tertiary amine/sulfinic acid chemically curing was developed (Kwon et al., 2012). For dual-cure luting, a chemically curing accelerator, based on aromatic sodium-sulfinate salts, is supplied in a separate/additional bottle with specific adhesives (Arrais et al., 2009). Self-etch primer-assisted composite cements, such as Panavia 21/F/F2.0 (Kuraray Noritake, Tokyo, Japan), cure using the BPO/tertiary amine/sulfinic acid system. These cements require the prior application of ED Primer (Kuraray Noritake), which separates the acidic monomers in Primer A from the aromatic sodium sulfinate coinitiator in Primer B, so enabling to produce sufficient free radicals for polymerization. Panavia F applied with ED Primer resulted in faster polymerization and higher DC than when applied without primer and light-curing. While this BPO/tertiary amine/sulfinic acid system works 
efficiently, the acidic functional monomers still neutralized the tertiary amines and resulted in lower DC (Kwon et al., 2012).

An alternative curing approach is being referred to as 'touch cure' or 'contact cure' (Kawano et al., 2015; Aung et al., 2019), by which polymerization starts at the cement-tooth interface. The commercial composite cement Panavia V5 (Kuraray Noritake) employs touch cure and contains a proprietary non-tertiary amine accelerator, which accelerates curing when it contacts the cement (Kuraray Noritake Panavia V5 technical information). However, the curing characteristics at actual cement-dentin interfaces have not profoundly been investigated.

To investigate the reaction mechanism of touch cure, we compared the effect of the accelerator-containing Panavia V5 Tooth Primer versus that of an experimental acceleratorfree primer on DC and shear bond strength (SBS) to dentin. Furthermore, the accelerator composition was assessed by X-ray fluorescence analysis (XRF), while the interfacial interaction with dentin was characterized correlatively by X-ray diffraction (XRD), 3D focused-ion-beam SEM and TEM. The null hypotheses tested were that (1) the accelerator in the tooth primer (TP) did not affect DC, and (2) this accelerator did not affect SBS to dentin.

\section{Materials and methods}

The commercial self-etch primer-assisted composite cement Panavia V5 was applied with (1) its commercial accelerator-containing Panavia V5 Tooth Primer ('comTP_acc'; pH=2.0), (2) an experimental accelerator-free primer, having the same composition without accelerator ('expTP_acc-free'; pH=2.0), and (3) without primer ('primer'). Additionally, the composite cement was either light-cured ('LIGHT') or left to chemically cure ('CHEM'), totaling to six experimental conditions. The full methodological detail and study design are schematically summarized in Fig. 1 and detailed in the Appendix. 
$D C$

The polymerization kinetics were monitored by real-time Fourier-transform infrared spectroscopy (RT-FTIR) on a Vertex 70 instrument (Bruker Optics). At 30 min, DC was quantified by attenuated total reflection (ATR)-FTIR.

$X R F$

Chemical elemental analysis of comTP_acc, expTP_acc-free and the Panavia V5 Pastes A and B was conducted using XRF (Rigaku ZSX Primus II, Rigaku, Tokyo, Japan).

SBS

Per experimental group, 10 specimens were subjected to SBS after having been stored dry for $30 \mathrm{~min}$ at $37^{\circ} \mathrm{C}$, as further detailed in the Appendix following our previous study (Yoshihara et al., 2020a).

$X R D$

Dentin surfaces $\left(8 \times 8 \mathrm{~mm}^{2}\right)$ of six molars were ground with \#600 SiC paper and treated with TP, a 15wt\% 10-MDP/ethanol/water solution ('10-MDP'), or left untreated prior to being examined by thin-film (TF)-XRD using a RINT2500 instrument (Rigaku, Tokyo, Japan) at an acceleration voltage of $40 \mathrm{kV}$, current of $200 \mathrm{~mA}$ and scanning rate of $0.02 \%$ with the angle of the X-ray beam fixed at $1.0^{\circ}$.

\section{FIB-SEM and TEM}

Dentin exposed at four additional molars were wet sanded using \#600 SiC paper, upon which TP and the composite cement were applied following the manufacturer's instructions. The cement surface was covered with a plastic strip (3M Oral Care, St. Paul, MN, USA) prior to being light-cured for 20s. Epoxy resin-embedded tooth specimens were observed by FIB-SEM 
(SMF-1000, HITACHI High-Tech Science, Tokyo, Japan) (Yoshihara et al., 2020b) and TEM (HRTEM, 200 kV; JEM-2100, JEOL).

\section{Results}

DC (Fig. 2)

RT-FTIR revealed that comTP_acc_LIGHT exhibited the fastest DC rate and highest DC. Confirming the RF-FTIR data, the highest 30-min DC was measured by ATR-FTIR for comTP_acc_LIGHT, being significantly higher than DC of expTP_acc_CHEM, which on its turn was significantly higher than that of expTP_acc-free_LIGHT/CHEM and primer_LIGHT/CHEM, respectively.

XRF (Fig. 3a)

XRF of comTP_acc identified phosphate $(P)$ and vanadium $(V)$, with only $P$ detected within expTP_acc-free. Aluminum (Al), silicon (Si), P, sulfur (S), and barium (Ba) were detected in Panavia V5 Paste A and B, with zinc ( $\mathrm{Zn}$ ) and strontium (Sr) additionally being present in Paste A.

SBS (Fig. 3b)

ComTP_acc_LIGHT/CHEM revealed the highest SBS, while significantly lower SBS was recorded for expTP_acc-free_LIGHT, its SBS on its turn being significantly higher than expTP_accfree_CHEM's SBS. The SBS data were supported by the fracture analysis that revealed for the luting procedures involving the accelerator-free TP more 'adhesive interfacial failures', indicative of weaker bonding effectiveness. Less 'adhesive interfacial failures' and more 'mixed adhesive-cohesive failures' were recorded for the luting procedures involving the acceleratorcontaining TP. 
XRD (Fig. 4a)

XRD of dentin treated with comTP_acc exhibited three characteristic peaks at $2 \theta=2.52^{\circ}$ $(d=3.51 \mathrm{~nm}), 4.84^{\circ}(\mathrm{d}=1.82 \mathrm{~nm})$, and $7.16^{\circ}(\mathrm{d}=1.23 \mathrm{~nm})$, which were assigned to 10-MDP_Casalt nano-layering (Yoshihara et al., 2011, 2013, 2019; Yoshida et al., 2012).

FIB-SEM (Fig. 4b) and TEM (Fig. 5a,b)

FIB-SEM revealed tight interfacial contact of the composite cement with dentin. A hybrid layer of about $300-500 \mathrm{~nm}$ was formed with a filler-poor zone on top, separating the composite cement from the dentin surface. Solely few filler particles could be observed, while no large particles of several micrometers were present in this layer. TEM disclosed a submicron hybrid layer that still contained hydroxyapatite without residual surface smear. Nano-layered structures were detected immediately adjacent of the interface. The filler-poor-zone was larger for comTP_acc than for expTP_acc-free.

\section{Discussion}

Strong adhesion of the restoration to the tooth prep and tight sealing of the restoration margins are fundamental to ensure clinical longevity of restorations with low susceptibility to secondary caries. When a glass-ceramic restoration is adhesively luted using an primer/adhesive-assisted composite cement, the cement bulk will cure and subject polymerization-shrinkage stress to the adhesive's bond to dentin, which is commonly weaker than the bond to the hydrofluoric acid-etched and silanized glass-ceramic restoration side (Lührs et al., 2014; Soares et al., 2017). To overcome this weakness, a 'touch-cure' concept was developed to strengthen the cement-tooth interface (Aung et al., 2019). When the composite cement touches the beforehand applied TP, the contained accelerator chemically sets off 
polymerization at the cement-tooth interface, hereby strengthening the adhesive restorationcement-tooth bond. However, no studies have investigated touch cure that makes use of an accelerator-containing TP. Therefore, we comparatively analyzed the effect of an acceleratorcontaining TP with that of a similar but accelerator-free primer on DC and SBS to dentin, with composite-cement luting without primer having served as negative control. In short, the commercial accelerator-containing TP supplied with the composite cement revealed faster and higher polymerization efficiency as well as higher SBS onto dentin when compared to the experimental accelerator-free TP (and the control without primer), by which both hypotheses advanced in this study were rejected. While non-etchable zirconia-ceramic restorations are less commonly adhesively luted upon sandblasting the zirconia surface followed by $10-\mathrm{MDP}$ priming, this touch-cure concept is expected to be also beneficial for zirconia-ceramic restorations that are adhesively luted using a primer-assisted composite cement.

A rapid-scan RT-FTIR technique was used to monitor the polymerization kinetics of the composite cement applied with/without accelerator in TP. RT-FTIR can follow the kinetics of double-bond conversion with time and has before been used to elucidate polymerization mechanisms (Pynaert et al., 2013; Perrot et al., 2016). RT-FTIR also helped understanding the effect of oxygen or hydroperoxide inhibition on polymerization by monitoring profiled polymerization kinetics (Pynaert et al., 2013). It allowed explaining the catalytic reaction by monitoring the reduction in $\mathrm{CO}_{2}$ and catalyst over a time scale of several seconds (Sheng et al., 2016). Here, RT-FTIR scanning of each primer/composite cement sample was conducted every 0.08 s to monitor the polymerization kinetics of six experimental conditions in real time. Direct evidence was provided that the accelerator-containing primer (comTP_acc) initiated polymerization quicker than the experimental accelerator-free primer (expTP_acc-free), both in LIGHT and CHEM mode. In CHEM mode, polymerization was initiated more rapidly using comTP_acc. When the accelerator-free primer was used in CHEM mode (expTP_accfree_CHEM), the composite started polymerizing at almost the same time as without TP 
(primer_LIGHT/CHEM). These findings indicate that when the composite cement touches the accelerator-containing primer, not only better but also faster polymerization is achieved. This achievement earlier blocks water uptake from dentin through osmosis that would otherwise result in interfacial porosities weakening the cement-tooth interface (Lührs et al., 2014).

Based on manufacturer's technical information and the safety data sheet of Panavia V5 (https://www.kuraraynoritake.eu/en/downloads/) (Appendix Table), Panavia V5 Tooth Primer (comTP_acc) contains a proprietary non-amine reducing agent as accelerator. Using XRF, we detected a vanadium compound in the commercial primer but not in the experimental primer, suggesting that vanadium acts as accelerator for polymerization. Furthermore, Panavia V5 Paste A contains hydroperoxide as chemical cure initiator, instead of BPO, along with an accelerator for light curing, while Paste B contains camphorquinone as light-cure initiator along with an accelerator for chemical curing (Fig. 5b) (https://www.kuraraynoritake.eu/en/page/product/techspecs/panavia). When Pastes A and B are mixed, chemical polymerization is initiated gradually (relatively slowly) upon interaction of the chemical cure initiator and accelerator (Fig. 5c). When this mixture makes contact with the accelerator-containing primer, the touch-cure mechanism is triggered, by which the chemical cure accelerator in the primer reacts with the chemical cure initiator in the mixed cement, hereby initiating polymerization at the composite cement-tooth interface (Fig. 5c).

In our investigation, the accelerator-containing TP (comTP_acc) resulted in faster polymerization of the composite cement. When the primer, consisting of MMAs dissolved in water, was applied to the tooth surface, the solvent was evaporated by air drying. As XRF is conducted in vacuum, the composition of the primer without solvent was assessed, approximating the condition (clinically) reached after solvent evaporation by air drying. XRF detected $92.1 \% \mathrm{P}$ and $7.9 \% \mathrm{~V}$, indicating that the concentration of the vanadium-compound accelerator relatively increased after evaporation, by which the chemical initiator within the composite cement can easily interact with the accelerator to initiate/promote polymerization 
at the actual primer/cement-tooth interface. The detected P represents 10-MDP. Note that the composite cement itself chemically polymerizes relatively slowly, because sufficient working time should be provided for mixing and the actual restoration-seating/luting procedure.

Both the light-cure (actually dual-cure) and the (solely) chemical cure DC curves of the composite cement in contact with the accelerator-containing TP (comTP_acc_LIGHT/CHEM) revealed a curve flattening at the beginning of the reaction, the latter more pronounced for the chemical cure DC curve. The first curve part before the flattening most likely represents the reaction of the primer accelerator with the composite-cement initiator, in particular induced by the relatively highly concentrated accelerator in TP. The ATR diamond cell used in this study resulted in an infrared light penetration of $1.6 \mu \mathrm{m}$ into the sample (https://www.bruker.com/fileadmin/user_upload/8-PDF-Docs/OpticalSpectrospcopy/FT-

IR/ALPHA/AN/AN79_ATR-Basics_EN.pdf). As the primer's film thickness is less than $1 \mu \mathrm{m}, \mathrm{FTIR}$ not only recorded polymerization at the primer-cement interface but also within the most adjacent composite cement. The second curve part after the curve flattening must then represent the cement's polymerization. The fact that the curve flattening was still recorded in the light-cure DC curve, means that the chemical touch cure at the primer-cement interface preceded the light-initiated polymerization. This time perspective must have helped stabilizing the bond of the primer/cement to the tooth during the ongoing polymerization of the composite cement. As well known, more rapid and intense polymerization is achieved when the composite cement is additionally irradiated with light (Jandt and Mills, 2013).

RT-FTIR uses a mercury cadmium telluride (MCT) detector, which does not enable fullquantitative element analysis due to a non-linear response (Shao and Griffiths, 2008). Therefore, ATR-FTIR was additionally employed to fully quantitatively determine DC at 30 min. When the composite cement was cured in contact with the accelerator-containing TP (comTP_acc), 30-min DC was significantly higher than DC of composite cement cured in contact with the experimental accelerator-free TP (expTP_acc-free) and cured without TP 
(primer). Light-curing (actually dual-curing) resulted in higher DC than when the composite cement was solely allowed to chemically cure, this however only significantly different when the composite cement was cured in contact with the commercial accelerator-containing TP (comTP_acc). These data correspond with previous research revealing higher DC when dualcure self-adhesive and primer/adhesive-assisted composite cements were additionally lightcured versus solely allowed to chemically cure (Moraes et al., 2011). The higher DC measured for comTP_acc_CHEM than for expTP_acc-free_CHEM must obviously be attributed to the non-amine vanadium compound chemical-cure accelerator present in TP. Comparing the use of the commercial and experimental TP (com/expTP), both containing 10-MDP as functional monomer, versus when no primer was employed (primef), the higher DC measured with primer confirms that the acidic functional monomer 10-MDP did not inhibit polymerization.

In literature, not much data correlating DC with bond strength is available. Our observations indicated that bond strength was in accordance with DC, as was also concluded before (Novais et al., 2017). Investigating a potential relationship of DC with 24-h micro-SBS revealed that dual-cure of the composite cements Variolink II (Ivoclar Vivadent) and RelyX ARC (3M Oral Care) exhibited higher DC and bond strength than those of the light-cure composite cement Variolink Veneer (Ivoclar Vivadent). Compared to dual-cure activation, DC of the solely lightcure composite cement was hence lower, with also differences in cement composition having influenced this outcome. Carvalho et al. (2017) measured the 24-h bond strength of the primer-assisted composite cement Multilink (Ivoclar Vivadent) and self-adhesive cement Clearfil SA Cement (Kuraray Noritake). Bond strength of both cements was lower when solely cured chemically, as opposed to being cured in dual-cure mode, thus also including light curing (Carvalho et al., 2017). Nevertheless, Multilink presented with a higher bond strength than Clearfil SA Cement, which should be explained by difference in composition but also by the presence of the functional monomer 10-MDP in Clearfil SA Cement. Acidic functional monomers in adhesives or self-adhesive cements were documented to reduce the efficacy of 
tertiary amines, used as accelerator for light- and chemically initiated polymerization (Oguri et al., 2012). Aguiar et al. (2015) evaluated the relationship between DC and mechanical properties, such as biaxial flexural strength/modulus. They studied two primer/adhesiveassisted composite cements, RelyX ARC (3M Oral Care) and Clearfil Esthetic Cement (Kuraray Noritake), and two self-adhesive composite cements, RelyX Unicem (3M Oral Care) and Clearfil SA Cement. DC of the self-adhesive composite cements at 20 min was lower in chemical cure and dual-cure mode than that of the primer/adhesive-assisted composite cements. The biaxial flexural strength and flexural modulus of the composite cements was higher when cured in dual-cure mode than in solely chemical cure mode. Obviously, the difference in composition between the cements investigated must also have influenced their mechanical properties.

In this study, only one cement was involved, by which the effect of compositional difference on SBS was excluded. Both the experimental groups using TP (com/expTP) yielded a higher bond strength with/without light-curing than when no primer was used. Moreover, the application of the experimental accelerator-free primer revealed higher SBS when the composite cement was light-cured versus solely allowed to chemically cure. Among all the tested groups, those solely allowed to chemically cure resulted in the lowest SBS. Lightactivation speeded up polymerization (Aguiar et al., 2015), and the rapid touch-cure polymerization of TP at the tooth-cement interface boosted DC and SBS.

The TPs studied (com/expTP) contained the acidic functional monomer 10-MDP, today considered as the most effective functional monomer, which is known (1) to relatively strongly etch providing surface micro-retention, (2) to ionically bind with hydroxyapatite within the submicron hybrid layer at dentin, and (3) to form stable self-assembled 10-MDP_Ca salts (Yoshihara et al., 2018). XRD confirmed the three peaks characteristic of 10-MDP_Ca-salt nanolayering upon treatment of dentin with comTP_acc (Yoshihara et al., 2010, 2011; Yoshida et al., 2012). Furthermore, the FIB-SEM 3D-reconstructed cement-dentin interface was tight, exhibiting a submicron hybrid layer at the interface with dentin. While the composite-cement 
bulk was highly filled with particles of different sizes, a 1-2 $\mu \mathrm{m}$ particle-free layer separated the composite cement from dentin. This resin-rich layer represents the primer that upon polymerization formed a separate layer thanks to touch cure, being larger than that of expTP_acc-free. TEM confirmed the submicron hybrid layer underneath the filler-free/poor layer. High-magnification TEM also confirmed 10-MDP_Ca-salt nano-layering produced at the composite cement-dentin interface. This morphologic interfacial feature can be considered as (indirect) evidence of chemical interaction of the functional monomer 10-MDP, included in TP, with hydroxyapatite, known to contribute to bond durability (Yoshihara et al., 2010, 2011, 2018; Yoshida et al., 2012). Adequate polymerization and thus high DC of the tooth-primer layer contributes to cement-dentin interfacial strength and stability.

Translated to clinical practice, when adhesively luting indirect restorations to preps of vital teeth in light of semi-direct (chairside one-visit) restorations, the adhesive is recommended to be cured separately and prior to the application of the composite cement (Lührs et al., 2014), obviously after thorough air-thinning not to hamper the restoration's fit. This clinical protocol stabilizes the adhesive-tooth interface and prevents/limits rapid water uptake through osmosis that would result in interfacial porosities and weaken the adhesive/cement-dentin bond. Alternatively and when adhesively luting indirect (two-visit) restorations, the adhesive is recommended to be applied and cured immediately using a so-called 'immediate dentin sealing' procedure (Magne et al., 2005; Frankenberger et al., 2007), this likewise to preserve the interface from rapid water sorption. The presently documented touch-cure mechanism using an initiator-containing TP, initiating polymerization at the actual interface with dentin, offers a good alternative to seal the interface at dentin without the need to separately lightcure an adhesive as part of adhesive luting using a primer/adhesive-assisted composite cement. Obviously, this touch-cure concept, initiating polymerization at the most sensitive interface with dentin, must also be favorable for other adhesive luting procedures, such as luting of zirconia-ceramic restorations and fiber posts. 
In conclusion, the touch-cure concept was investigated using real-time DC monitoring along with bond-strength testing. The initiator-containing TP cured quickly at the interface with dentin and strengthened the bond as compared to the experimental initiator-free primer. Polymerization initiation at the actual interface with dentin better resists de-bonding due to polymerization shrinkage, while also blocks rapid water uptake that weakens the bond (Lührs et al., 2014a,b).

\section{Author Contributions}

Yoshihara $\mathrm{K}$, contributed to conception, design, data acquisition, analysis and interpretation, drafted and critically revised the manuscript; Nagaoka N, contributed to conception, design, data acquisition, analysis and interpretation, and critically revised the manuscript; Benino $\mathrm{Y}$, contributed to conception, design, data acquisition, analysis and critically revised the manuscript; Nakamura A, contributed to conception, design, data acquisition, analysis and critically revised the manuscript; Hara T, contributed to conception, design, data acquisition, analysis and critically revised the manuscript; Maruo Y, contributed to conception, design, data acquisition, analysis and critically revised the manuscript; Yoshida $\mathrm{Y}$, contribute to conception, interpretation, and critically revised the manuscript; Van Meerbeek B, contribute conception, interpretation, drafted and critically revised the manuscript. All authors gave final approval and agree to be accountable for all aspects of the work.

\section{Acknowledgments}

This work was supported by the NIMS microstructural characterization platform as part of the "Nanotechnology Platform" of the Ministry of Education, Culture, Sports, Science and Technology (MEXT) of Japan. This study was additionally supported by JSPS KAKENHI with the grant number JP 18K17068. The authors declare that they have no conflict of interest in this 
research. We thank Kuraray Noritake for providing the experimental tooth primer formulation. 


\section{References}

Achilias DS, Sideridou ID. 2004. Kinetics of the benzoyl peroxide/amine initiated free-radical polymerization of dental dimethacrylate monomers: Experimental studies and mathematical modeling for TEGDMA and Bis-EMA. Macromolecules 37(11):4254-4265.

Aguiar TR, de Oliveira M, Arrais CA, Ambrosano GM, Rueggeberg F, Giannini M. 2015. The effect of photopolymerization on the degree of conversion, polymerization kinetic, biaxial flexure strength, and modulus of self-adhesive resin cements. J Prosthet Dent. 113(2):12834.

Arrais CAG, Giannini M, Rueggeberg FA. 2009. Effect of sodium sulfinate salts on the polymerization characteristics of dual-cured resin cement systems exposed to attenuated light-activation. J Dent. 37(3):219-227.

Aung SSMP, Takagaki T, Ko AK, Halabi S, Sato T, Ikeda M, Nikaido T, Burrow MF, Tagami J. 2019. Adhesion durability of dual-cure resin cements and acid-base resistant zone formation on human dentin. Dent Mater. 35(7):945-952.

Burker Optics, Technical information, Application Note AN \# 79: Attenuated Total Reflection (ATR), a versatile tool for https://www.bruker.com/fileadmin/user_upload/8-PDF-Docs/OpticalSpectrospcopy/FTIR/ALPHA/AN/AN79_ATR-Basics_EN.pdf.

Carvalho AA, Stefani A, de Sá Barbosa WF, Lopes LG, Giannini M. 2016. Influence of curing mode of resin luting cements on bond strength to dentin. Braz J Oral Sci. 15(4):258-263.

De Souza G, Braga RR, Cesar PF, Lopes GC. 2015. Correlation between clinical performance and degree of conversion of resin cements: a literature review. J Appl Oral Sci. 23(4):358-368.

Frankenberger R, Lohbauer U, Taschner M, Petschelt A, Nikolaenko SA. 2007. Adhesive luting revisited: influence of adhesive, temporary cement, cavity cleaning, and curing mode on internal dentin bond strength. J Adhes Dent. 9(Suppl 2):269-73.

Jandt KD, Mills RW. 2013. A brief history of LED photopolymerization. Dent Mater. 29(6):605- 
617.

Kawano S, Fu J, Saikaew P, Chowdhury AFMA, Fukuzawa N, Kadowaki Y, Kakuda S, Hoshika S, Nakaoki Y, Ikeda T, Tanaka T, Sano H. 2015. Microtensile bond strength of a newly developed resin cement to dentin. Dent Mater J. 34(1):61-69.

Kuraray Noritake Panavia V5 technical information: https://kuraraydental.com/wpcontent/uploads/2018/05/panavia-v5-technical-information.pdf.

Kwon TY, Bagheri R, Kim YK, Kim KH, Burrow MF. 2012. Cure mechanisms in materials for use in esthetic dentistry. J Investig Clin Dent. 3(1):3-16.

Lührs AK, De Munck J, Geurtsen W, Van Meerbeek B. 2014a. Composite cements benefit from light-curing. Dent Mater. 30(3):292-301.

Lührs AK, Pongprueksa P, De Munck J, Geurtsen W, Van Meerbeek B. 2014b. Curing mode affects bond strength of adhesively luted composite CAD/CAM restorations to dentin. Dent Mater. 30(3):281-91.

Magne P, Kim TH, Cascione D, Donovan TE. 2005. Immediate dentin sealing improves bond strength of indirect restorations. J Prosthet Dent. 94(6):511-9.

Moraes RR, Boscato N, Jardim PS, Schneider LF. 2011. Dual and self-curing potential of selfadhesive resin cements as thin films. Oper Dent. 36(6):635-642.

Novais VR, Raposo LHA, de Miranda RR, Lopes CDCA, Simamoto Júnior PC, Soares CJ. 2017. Degree of conversion and bond strength of resin-cements to feldspathic ceramic using different curing modes. J Appl Oral Sci. 25(1):61-68.

Nagaoka N, Yoshihara K, Feitosa VP, Tamada Y, Irie M, Yoshida Y, Van Meerbeek B, Hayakawa S. 2017. Chemical interaction mechanism of 10-MDP with zirconia. Sci Rep. 7(1):1-7.

Oguri M, Yoshida Y, Yoshihara K, Miyauchi T, Nakamura Y, Shimoda S, Hanabusa M, Momoi Y, Van Meerbeek B. 2012. Effects of functional monomers and photo-initiators on the degree of conversion of a dental adhesive. Acta Biomater. 8(5):1928-34.

Perrot D, Croutxé-Barghorn C, Allonas X. 2016. UV-curable thio-ether-urethane network with 
tunable properties. J. Polym. Sci. A 54(19):3119-3126.

Pynaert R, Buguet J, Croutxé-Barghorn C, Moireau P, Allonas X. 2013. Effect of reactive oxygen species on the kinetics of free radical photopolymerization. Polym Chem. 4 (8): 2475-2479.

Shao L, Griffiths PR. 2008. Correcting nonlinear response of mercury cadmium telluride detectors in open path Fourier transform infrared spectrometry. Anal Chem. 80(13):52195224.

Sheng H, Frei H. 2016. Direct observation by rapid-scan FT-IR spectroscopy of two-electronreduced intermediate of tetraaza catalyst $\left[\mathrm{CollN}{ }_{4} \mathrm{H}(\mathrm{MeCN})\right]^{2+}$ converting $\mathrm{CO}_{2}$ to $\mathrm{CO}$. J Am Chem Soc. 138(31):9959-9967.

Soares CJ, Faria-E-Silva AL, Rodrigues MDP, Vilela ABF, Pfeifer CS, Tantbirojn D, Versluis A. 2017. Polymerization shrinkage stress of composite resins and resin cements - What do we need to know? Braz Oral Res. 31(suppl 1):e62.

Yoshida Y, Yoshihara K, Nagaoka N, Hayakawa S, Torii Y, Ogawa T, Osaka A, Meerbeek BV. 2012. Self-assembled nano-layering at the adhesive interface. J Dent Res. 91(4):376-81.

Yoshida Y, Nagakane K, Fukuda R, Nakayama Y, Okazaki M, Shintani H, Inoue S, Tagawa Y, Suzuki K, De Munck J, Van Meerbeek B. 2004. Comparative study on adhesive performance of functional monomers. J Dent Res. 83(6):454-458.

Yoshihara K, Yoshida Y, Nagaoka N, Fukegawa D, Hayakawa S, Mine A, Nakamura M, Minagi S, Osaka A, Suzuki K, Van Meerbeek B. 2010. Nano-controlled molecular interaction at adhesive interfaces for hard tissue reconstruction. Acta Biomater. 6(9):3573-3582.

Yoshihara K, Yoshida Y, Hayakawa S, Nagaoka N, Irie M, Ogawa T, Van Landuyt KL, Osaka A, Suzuki K, Minagi S, Van Meerbeek B. 2011. Nanolayering of phosphoric acid ester monomer on enamel and dentin. Acta Biomater. 7(8):3187-3195.

Yoshihara K, Yoshida Y, Nagaoka N, Hayakawa S, Okihara T, De Munck J, Maruo Y, Nishigawa G, Minagi S, Osaka A, Van Meerbeek B. 2013. Adhesive interfacial interaction affected by different carbon-chain monomers. Dent Mater. 29(8):888-897. 
Yoshihara K, Hayakawa S, Nagaoka N, Okihara T, Yoshida Y, Van Meerbeek B. 2018. Etching efficacy of self-etching functional monomers. J Dent Res. 97(9):1010-1016.

Yoshihara K, Nagaoka N, Yoshida Y, Van Meerbeek B, Hayakawa S. 2019. Atomic level observation and structural analysis of phosphoric-acid ester interaction at dentin. Acta Biomater. 97:544-556.

Yoshihara K, Nagaoka N, Maruo Y, Nishigawa G, Yoshida Y, Van Meerbeek B. 2020a. Silanecoupling effect of a silane-containing self-adhesive composite cement. Dent Mater. 36(7):914-926.

Yoshihara K, Nagaoka N, Nakamura A, Hara T, Hayakawa S, Yoshida Y, Van Meerbeek B. 2020b. Three-dimensional observation and analysis of remineralization in dentinal caries lesions. Sci Rep. 10(1):1-10. 


\section{Figure legends}

Figure 1. Schematic diagram explaining the experimental study set-up.

Figure 2. DC of the composite cement applied following the six experimental conditions, as measured in real time in rapid-scan mode using RT-FTIR in (a) and by conventional ATR-FTIR 30 min after application in (b). (a) The second highest DC was achieved by comTP_acc_CHEM, while its DC/time curve revealed after a rapid initial increase a temporally reduced DC rate (curve flattening) to next re-accelerate and reach a DC plateau being lower than that reached by comTP_acc_LIGHT. The latter presented with a similar but much smaller/shorter curve flattening. This curve flattening at the polymerization beginning was not recorded for expTP_acc-free_LIGHT/CHEM, while DC of primer_LIGHT/CHEM was much slower to reach the lowest DC plateau recorded. ExpTP_acc-free_LIGHT reached a DC plateau in between that recorded for comTP_acc_CHEM and the almost equal plateaus reached by expTP_accfree_CHEM and primer_LIGHT/CHEM. (b) The bars represent the average 30-min DC of the six experimental groups as fully quantified by ATR-FTIR. Different letters indicate statistically significant difference $(p<0.05)$.

Figure 3. Chemical composition of the different materials/products used in this study as analysed by XRF in (a), and shear-bond strength (SBS) to dentin of the composite cement applied following the four experimental groups involving the application of the acceleratorcontaining ('comTP_acc') and accelerator-free ('expTP_acc-free') tooth primer applied in 'LIGHT' and 'CHEM' mode in (b).

Figure 4. XRD of dentin treated with the accelerator-containing tooth primer ('comTP_acc') and the experimental 10-MDP water/ethanol solution ('10-MDP'), as well as of untreated dentin in (a), and FIB-SEM 3D reconstruction of the cement-tooth interface produced by the 
self-etch primer-assisted composite cement Panavia V5 applied with Tooth Primer in (b). FIBSEM revealed a tight interfacial contact of the composite cement with dentin. FIB-SEM images were obtained by mixed backscattered and secondary electron imaging. The bright contrast at the hybrid layer should be attributed to intensive secondary electron emission at the heterogenous interface. The 3D reconstruction additionally showed that the composite cement contains filler particles of different sizes. Separating the composite cement from dentin, a 1-2 $\mu \mathrm{m}$ particle-poor layer represents the primer layer that polymerized by the touchcure mechanism.

Figure 5. TEM photomicrographs of the composite cement-dentin interface produced by Panavia V5 applied with Tooth Primer (comTP_acc) at different magnifications in (a), applied with the experimental accelerator-free tooth primer (expTP_acc-free) in (b), and schematic explaining the 'touch-cure' polymerization reaction before and after polymerization in (c). TEM disclosed a 500-nm hybrid layer that still contained hydroxyapatite without structural indications of residual surface smear $(a, b: A-C)$. The submicron hybrid layer corresponds to the transition zone observed between the filler-poor zone and dentin at the 3D-reconstructed cement-dentin interface. This filler-poor-zone was larger for comTP_acc $(a: A, B)$ than for expTP_acc-free (b: A,B). High-magnification TEM revealed nano-layered structures immediately adjacent of the interface $(a, b: D)$. 


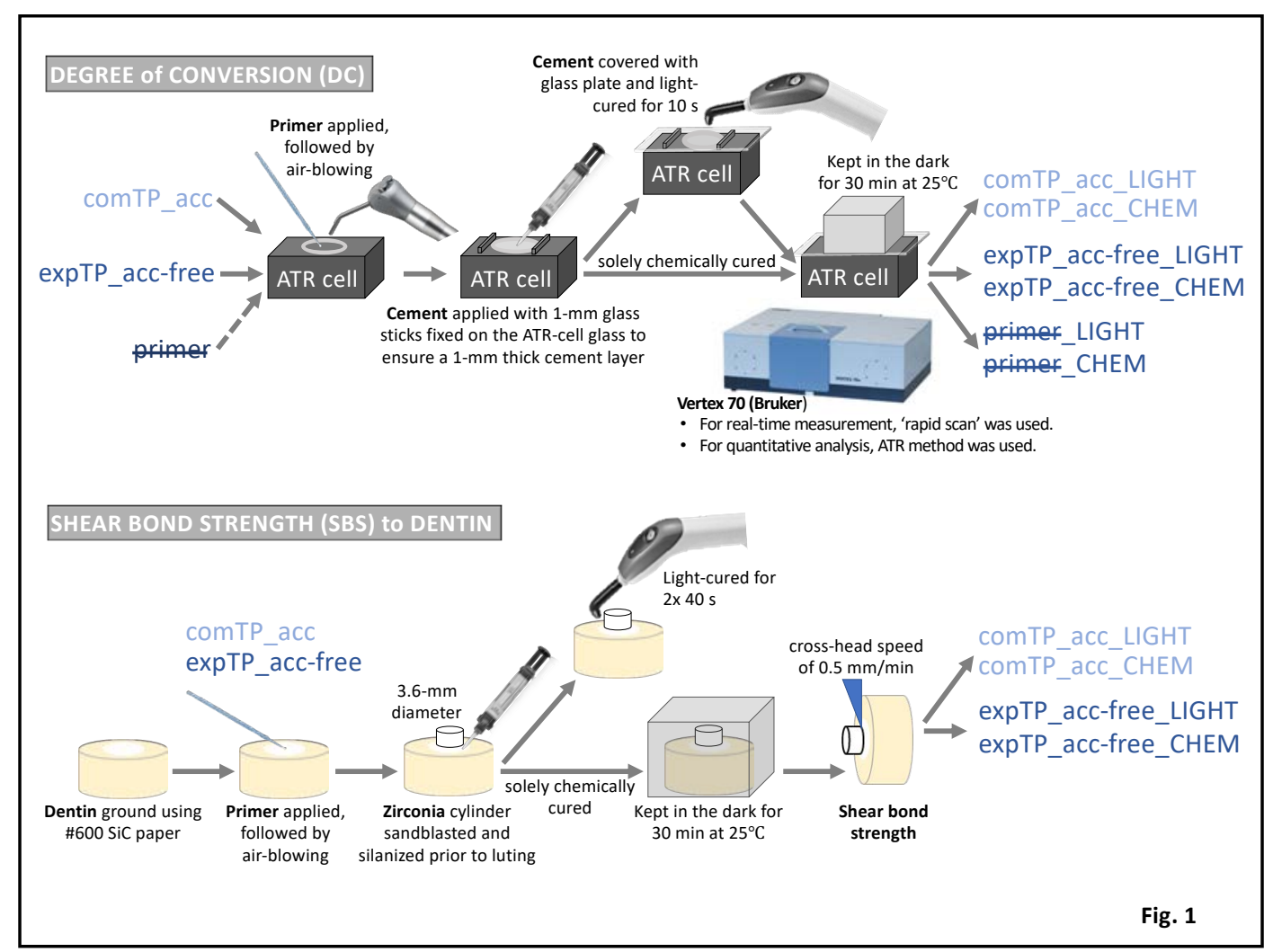

1

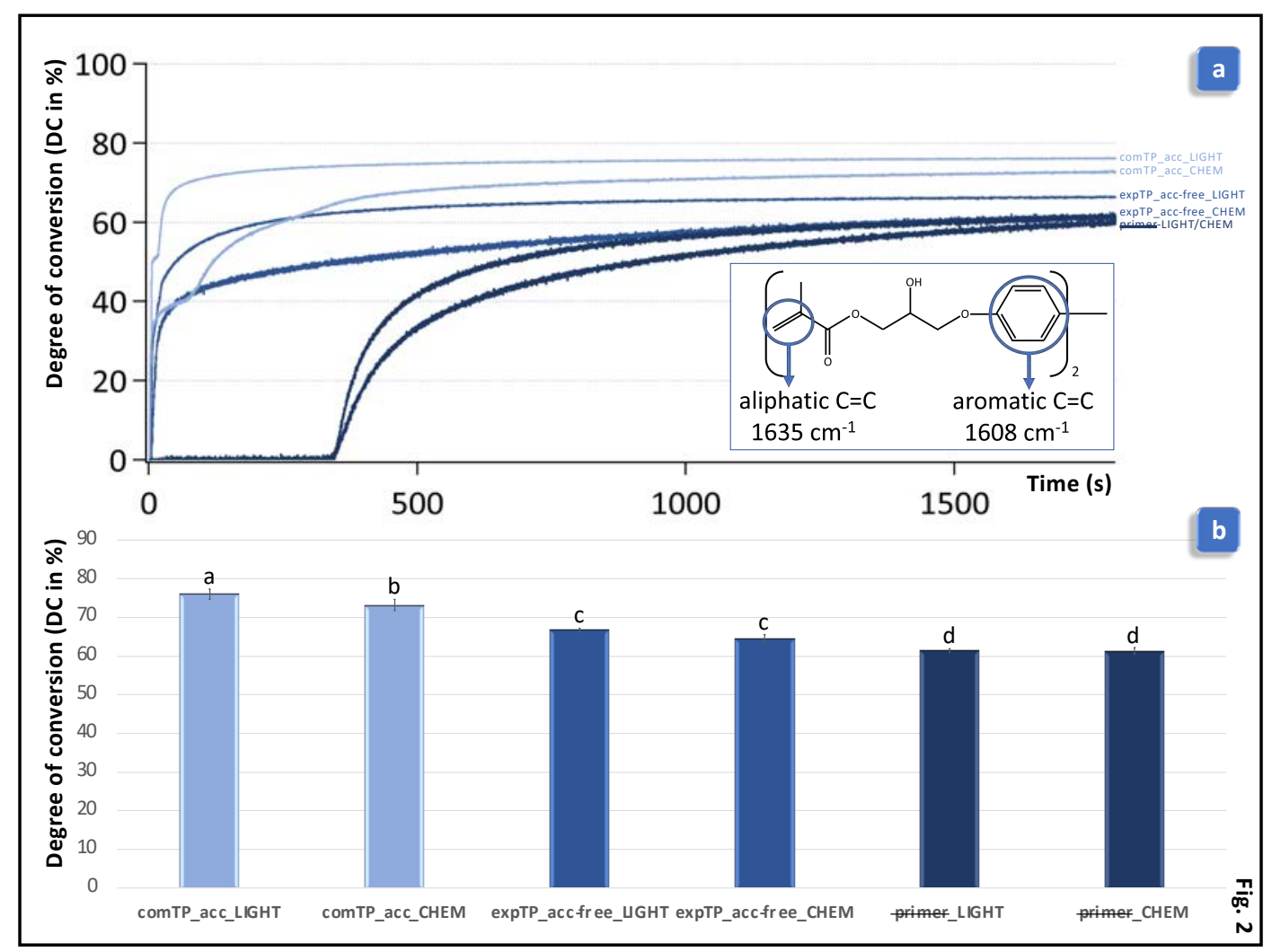

2 


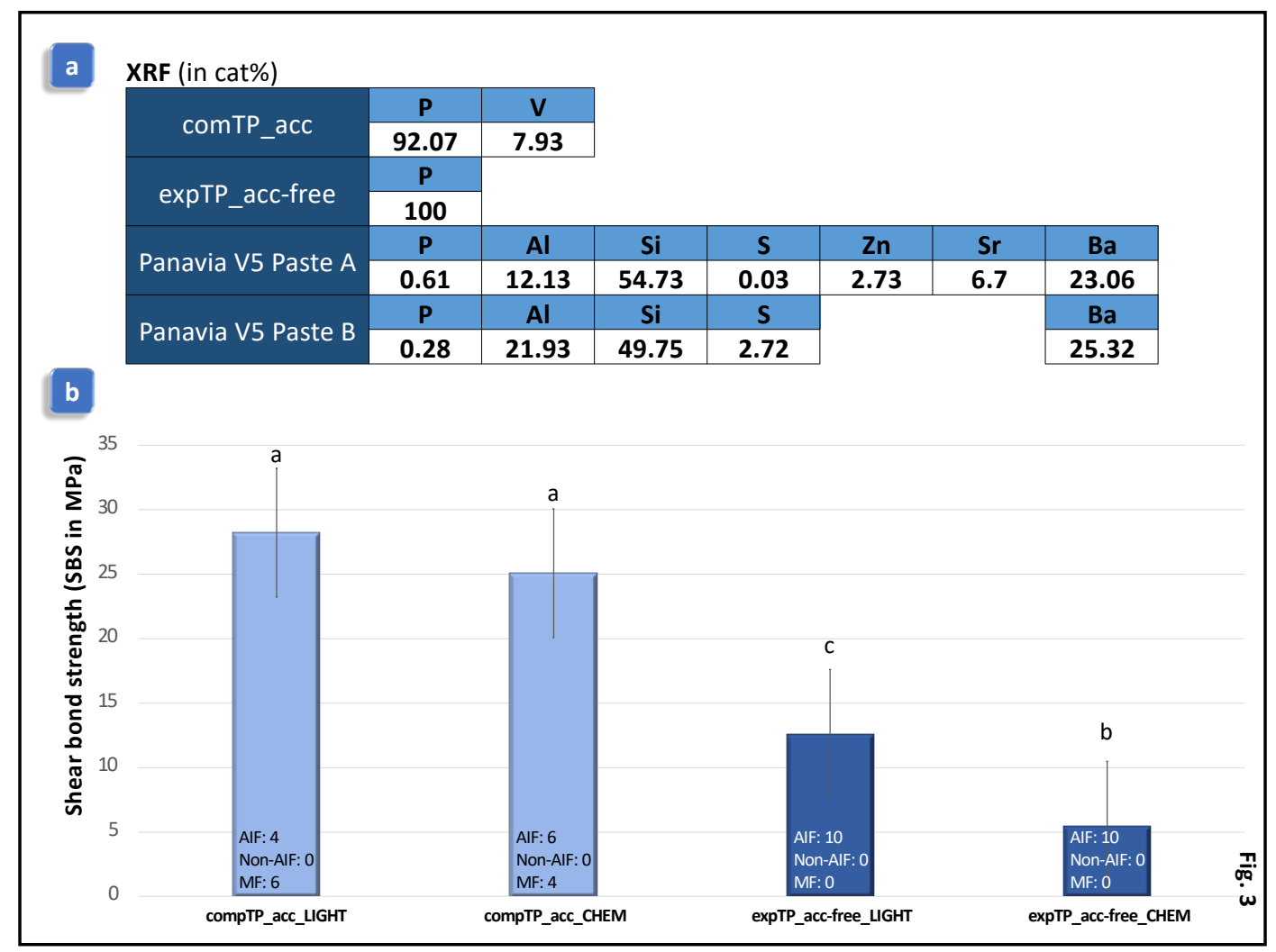

3

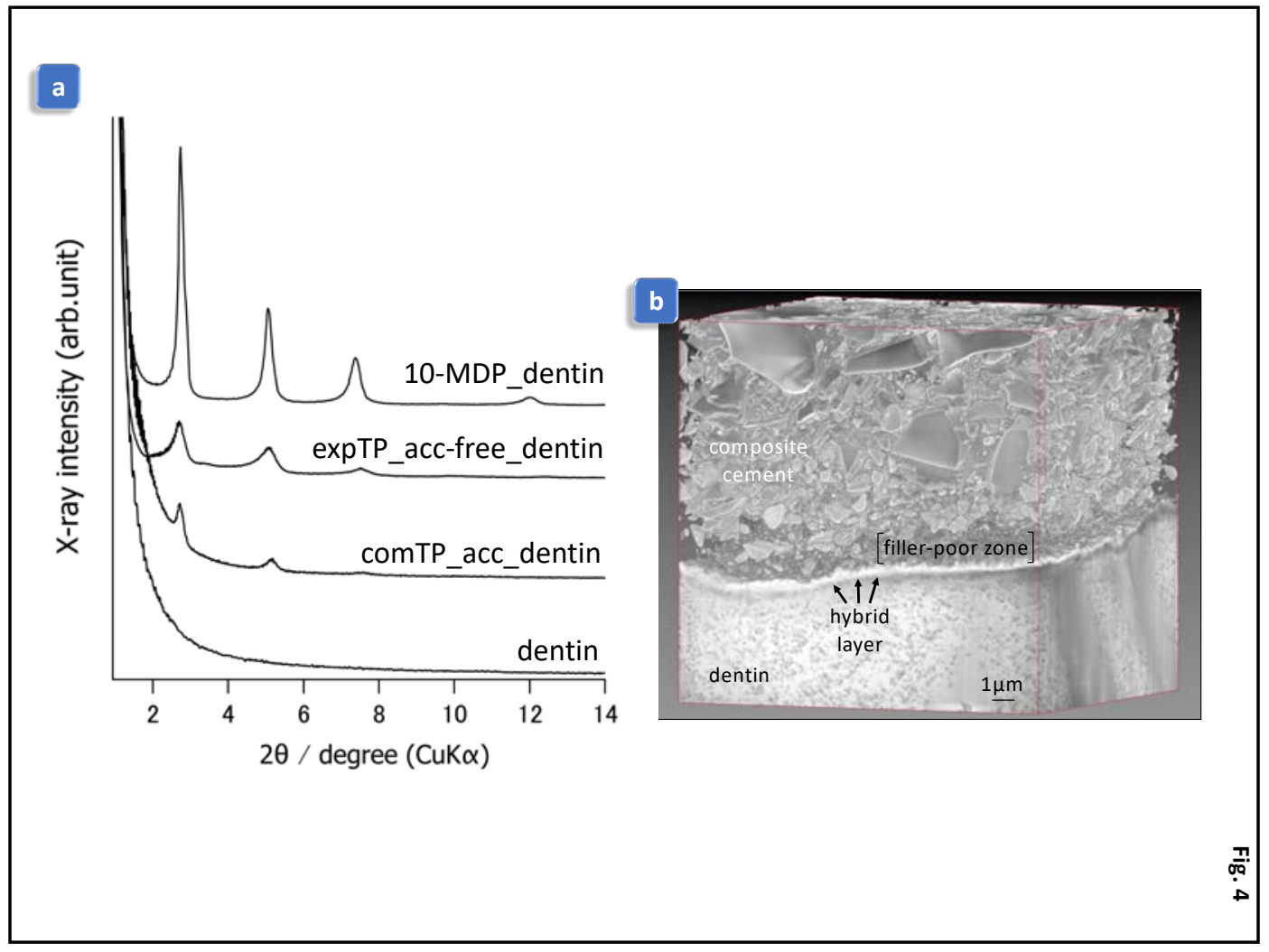




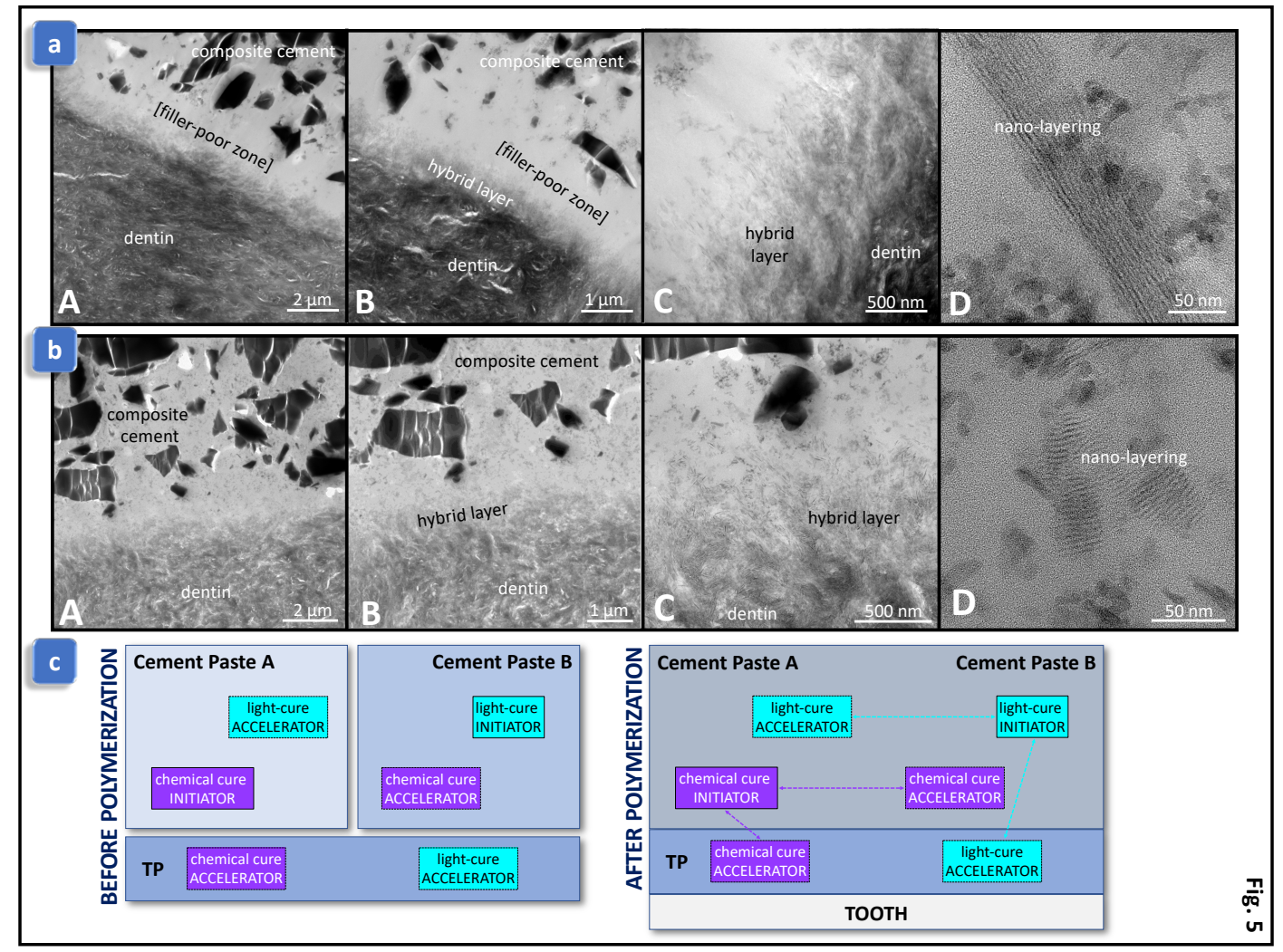

5 